\title{
Some Thoughts on Logic and Intuition in Science and Chemical Engineering
}

\author{
Christo Boyadjiev \\ Institute of Chemical Engineering, Bulgarian Academy of Sciences, Sofia, Bulgaria \\ Email: chboyadj@bas.bg
}

Received 8 July 2014; revised 12 August 2014; accepted 18 September 2014

Copyright (C) 2014 by author and OALib.

This work is licensed under the Creative Commons Attribution International License (CC BY). http://creativecommons.org/licenses/by/4.0/

(c) (i) Open Access

\begin{abstract}
The role of the logic and intuition in the chemical engineering is presented. Basic rules of logic in chemical engineering are used: "Construction of mathematical models of chemical engineering structures use the general rules (approximations) of the continuous media mechanics" and "A full correspondence between physical effects and mathematical operators (in the form of functions and their derivatives) in the mathematical models". The basic mathematical operators as gradient, divergence and Laplace operator are associated with the physical effects in the models. The intuition-judgments in the chemical engineering are the axioms as "Stokes postulate for the linear relationship between stress and strain rate", "linear relationship between the diffusion flux and the concentration gradient (Fick's first law)", "linear relationship between heat flux and temperature gradient (Fourier's first law)". The intuition-judgments (axioms) of the linear and nonlinear mass transfer theories are presented too.
\end{abstract}

\section{Keywords}

Chemical Engineering, Logic, Intuition-Judgment, Intuition-Hunch, Axiom, Theorem

Subject Areas: Chemical Engineering \& Technology, Theoretical Chemistry

\section{Introduction}

The logic and intuition form the human knowledge. They are inextricably interlinked and form the basis of the science, arts and religions, with the differences that in the science the explanations are dominated by logic based on physical laws and consequent developed relationships, while in the art and the religions the intuition and the formal logic explain the real world [1].

The logic is a combination of classical (formal) logic, which uses a set of rules, laws, and "non-classical logics" (rules which are valid for specific situations). The classical logic uses as a basic rule "the law of excluded middle": i.e., every statement is either true or false. In this context, the classical physics of this type of logic 
forms the basis of scientific analyzes. However, the electron motion explanation, for instance, requires using an additionally "chance" (that is, a proposition is true with a specified degree of probability). The formal logic leads to conclusions, which are historically invariable and easily observable in any religion. In the classical science (mainly in the physics) this approach allows new (additional) laws (rules) to be conceived without any effects on the old ones.

The intuition has many forms: intuition-judgment, allegation (discretion, judgment of the intuitive truth), intuition-hunch, presentiment (heuristic intuition, assumption, expectation, anticipation) and insight-insight (from experience). The intuition-judgment is a direct discretion of the truth that needs no proof. In mathematics, intuition-judgment is the axiom. The intuition-hunch, is a subject to a proof theoretically (mathematically, logically) or experimentally. In mathematics, the intuition-hunch is the theorem.

\section{Logic and Intuition in Science}

The logic and intuition in the natural sciences are always interrelated; however, the logic has the advantage to generate a consequent chain of rules. The mathematics, the geometry for instance, is a perfect example of interaction between logic and intuition, where they are clearly differentiated. The logic of Euclidean geometry is based on axioms (intuitions-judgments). For example: "Cross a point not on the line, you can draw one parallel (disjoint) straight only”. Axiomatic framework can be extended (Riemann or Lobachevsky geometry). In some areas of the mathematics a deductive approach is used, i.e. previously formulated definitions and axioms are the basis of a strictly logical proof of new propositions (theorems). In this approach, the problems of mathematical logic are related with the internal consistency (uncontradiction) of the definitions and axioms, and a sufficiency of the completeness for the problem solution (solvability).

In the natural sciences the intuition-judgment plays the role of axioms and is the basis of the laws (theories), but they can be complemented (developed). For example: Newtonian mechanics, the physics of FaradayMaxwell, the theory of relativity, the quantum mechanics. The intuition-hunch (guesswork) in these sciences is possible to be corroborated experimentally too, however, the number of experiments is always limited. If 100 experiments corroborate, for instance, the there is no guarantee that the next 101 experiment will not be rejected, i.e. the experimental verification is intuition-hunch. It should be noted that the practice is not a "criterion of truth" because it is only a "test of truth", because the practice (experiment) can only reject false judgments, but experimentally it is practically impossible to confirm the general loyalty of the true tested.

The scientific knowledge of the real world is based the formal logic with the experimental verification criteria and verdicts: "out of the logical" reasoning or "correct”. Such verdicts are enough for synthesis outputs (synthetic conclusions). This approach is more powerful than sole formal logic and presents the knowledge theory. It uses a discursive approach (a form of deductive approach), i.e. a formal-logical approach, which includes a new "out of the logical” judgments which have a character of axiom, if they are generally acknowledged (e.g, with experimental confirmation).

At the beginning of the last century (1934) the Great Godel's theorem (an outstanding achievement of mathematical logic) put an end of the unsuccessful attempts of mathematicians to neglect the role of intuition. It has been proven that a complete formalization of the mathematics (its transforming into a deductive theory) is impossible, as follows from the strict Godel's theorem: "Whatever initial set of axioms, definitions and rules to be set, always in the process development of the reasoning (in the development of the mathematics) will come to claim, for which (being limited by pre-formulated axiomatic basis) it can not say whether true or false”. To move forward it requires a new axiom (intuition-judgment).

The science development, as a result of a combination of logic and intuition, is described by an exponential function and shows that the number of scientists, the number of published scientific papers and the material costs for science grow, doubling approximately every 20 - 30 years, i.e. within the time of formation of a new generation [1].

If it compare with the "industrial revolution" (18 - 19 century), which freed man from the crude, standardized physical labor, the computerization (20 - 21 century) leads to "intellectual revolution”, freeing man from the "rough", a standardized (which is possible to be formalized), intellectual labor (from the rough logical operations). This leads to a change in the ratio of logic and intuition in science and technology, enhancing the intuitive component of creativity. 


\section{How the Logic and the Intuition Work in Chemical Engineering}

\subsection{Basic Rules and Relationships}

The chemical engineering as a science encompasses the mechanism and kinetics of the processes and systems in the chemical and other process industries with regard to their optimal practical implantation at varies stages of this course of action: the engineering design, management and renovation (reconstruction), etc. In this sense, the role of the logic and the intuition is related primarily to the science relevant to process mechanisms and kinetics as well as to the basic techniques allowing formalizing the created knowledge: modeling and simulation.

The logic of chemical engineering is a construction of rules and laws taken from the formal logic and as well as its own specific rules and laws. The specific components of the chemical engineering logic are mainly related to the stages of modeling, namely:

- Formulation of hypotheses about the mechanism of the process;

- Construction of mathematical structures in accordance with the modeled process mechanisms;

- Identification of parameters in the mathematical models using the experimental data;

- Statistical analysis of model adequacy.

Therefore, it is possible to formulate some basic rules of logic relevant to chemical engineering:

1) Construction of mathematical models of chemical engineering structures on the basis of general rules (approximations) using the mechanics of continua, where the mathematical point is replaced by a small (elementary) representative physical volume; sufficiently small with respect to the real volume of the device, but enough large with respect to the intermolecular volumes of the medium modelled. In a cylindrical columns, a common design of chemical devices, for instance, the elementary volume, for example, is a small cylinder with a radius, equal to that of the column and a height sufficiently small with respect to the column height, that is "a thin slice" with a cross-section area equal to that of the column. Further, all physical quantities in this elementary slice are constant values across its volume and can vary in time only.

2) Another general rule in the mathematical models in chemical engineering is a full correspondence between physical effects and mathematical operators (in the form of functions and their derivatives) describing them.

\subsection{Physical Effects and Mathematical Operators}

The particular rules of the logic in the chemical engineering are associated with the physical quantities in the models, which are scalars, vectors and tensors:

1) The scalars (density, pressure, concentration, temperature) are determined by a number (a constant value in the elementary volume):

$$
\rho=\rho(x, y, z, \tau), \quad p=p(x, y, z, \tau), \quad c=c(x, y, z, \tau), \quad t=t(x, y, z, \tau) .
$$

2) The vectors (force, velocity, acceleration) are determined by three numbers, which are the values of their components (their projections on the coordinate axes):

$$
\boldsymbol{u}=u_{x} \boldsymbol{i}+u_{y} \boldsymbol{j}+u_{z} \boldsymbol{k}, \quad u_{i}=u_{i}(x, y, z, \tau), \quad i=x, y, z .
$$

3) The tensors (stress tensor) are determined with the help of nine numbers:

$$
\Pi=\left(\begin{array}{ccc}
\sigma_{x} & \tau_{x y} & \tau_{x z} \\
\tau_{y x} & \sigma_{y} & \tau_{y z} \\
\tau_{z x} & \tau_{z y} & \sigma_{z}
\end{array}\right) .
$$

An orthogonal Cartesian co-ordinate system is considered in the model descriptions hereafter in this article.

It should be noted that the tension is a force exerted per unit area. It can be represented by its components (one normal and two tangential) with respect to that surface in a given co-ordinate system. Each of these components has three projections on the coordinate axes, i.e. the tension has a direction as a vector, but does not form a vector field and is defined by a tensor field.

Other special rules of the logic in the chemical engineering are associated with one correspondence between physical effects and mathematical operators gradient, divergence, Laplacian, etc.: 
1) Gradient. The scalars form a scalar field, where the direction of the largest changes is determined by the vector-gradient:

$$
\boldsymbol{g r a d} c=\nabla c=\frac{\partial c}{\partial x} \boldsymbol{i}+\frac{\partial c}{\partial y} \boldsymbol{j}+\frac{\partial c}{\partial z} \boldsymbol{k}
$$

2) Divergence. The vectors form a vector field. Vector flux through a closed surface of a elementary volume is the scalar-divergence:

$$
\operatorname{div} \boldsymbol{u}=\frac{\partial u_{x}}{\partial x}+\frac{\partial u_{y}}{\partial y}+\frac{\partial u_{z}}{\partial z}
$$

3) Laplace operator. The divergence of the gradient vector:

$$
\operatorname{div}(\operatorname{grad} c)=\frac{\partial u_{x}}{\partial x}+\frac{\partial u_{y}}{\partial y}+\frac{\partial u_{z}}{\partial z} .
$$

The own laws of the logic in the chemical engineering are the laws of conservation of mass and energy, and the Newton's second law [2]:

1) The conservation laws (continuity of the convective flow of volume, mass and energy) are obtained by equalization to zero the velocity divergence and the convective fluxes of the mass and energy, i.e. what is included in the elementary volume (due to fluid flow) is equal to what comes out:

$$
\operatorname{div} \boldsymbol{u}=0, \quad \operatorname{div}(\rho \boldsymbol{u})=0, \quad \operatorname{div}(\rho c \boldsymbol{u})=0, \quad \operatorname{div}(\rho \varepsilon \boldsymbol{u})=0 \quad\left(\varepsilon=c_{p} T+\varepsilon_{0}\right) .
$$

The presence of a difference between input and output offset by the changes in value over time:

$$
\frac{\partial \rho}{\partial t}+\operatorname{div}(\rho \boldsymbol{u})=0, \quad \frac{\partial}{\partial t}(\rho c)+\operatorname{div}(\rho c \boldsymbol{u})=0, \quad \frac{\partial}{\partial t}(\rho \varepsilon)+\operatorname{div}(\rho \varepsilon \boldsymbol{u})=0 .
$$

2) The Newton's second law-the product of mass per unit volume ( $\rho$ ), and full acceleration in space and time $(\boldsymbol{a})$ is equal to the sum of the forces acting per unit volume (volume force $\boldsymbol{K}$ and surface force $\boldsymbol{P}$ ):

$$
\rho \boldsymbol{a}=\boldsymbol{K}+\boldsymbol{P} .
$$

This law is valid in the elementary volume and is the basis of the laws of hydrodynamics laws.

\subsection{Intuition in Chemical Engineering}

The intuition-judgments in the chemical engineering are the axioms, which are the basis of the laws (linear theories), but they are possible to be developed (non-linear theories [3]). The laws of linear theories can not be checked experimentally, but only their effects.

The hydrodynamics of the Newtonian fluids use as an axiom of the Stokes postulate for the linear relationship between stress and strain rate, where the coefficient of proportionality is the coefficient of dynamic viscosity $(\mu)$. This leads to Newton's law (axiom for the Newtonian fluids):

$$
\tau=-\mu \frac{\partial u}{\partial n}
$$

On this basis, the Navier-Stokes equations are obtained. For non-Newtonian fluids a more complex rheological laws (axioms) must be used.

The mathematical description of the diffusion processes uses the axiom of the linear relationship between the diffusion flux and the concentration gradient (Fick's first law):

$$
q_{c}=-D \operatorname{grad} \rho c=-D \nabla \rho c,
$$

where the coefficient of proportionality is the diffusivity $(\boldsymbol{D})$. For some fluids the non-linear law must be used, i.e. $D=D(c)$. 
The participation of the diffusion and convective mass flux in the mass balance in the elementary volume leads to a convection-diffusion equation:

$$
\frac{\partial}{\partial t}(\rho c)+\operatorname{div}(\rho c \boldsymbol{u})+\operatorname{div} \boldsymbol{q}_{c}=0, \quad \frac{\partial}{\partial t}(\rho c)+\operatorname{div}(\rho c \boldsymbol{u})=\operatorname{div}[D \nabla(\rho c)] .
$$

The thermal processes use the axiom of a linear relationship between heat flux and temperature gradient (Fourier's first law):

$$
\boldsymbol{q}_{T}=-\lambda \operatorname{grad} T=-\lambda \nabla T
$$

where the coefficient of proportionality is the coefficient of thermal conductivity $(\lambda)$. For some liquids must be used a non-linear law $\lambda=\lambda(T)$. The participation of the heat flux due to conduction and convection in the heat balance in the elementary volume leads to an equation of convection-heat conduction:

$$
\frac{\partial}{\partial t}(\rho \varepsilon)+\operatorname{div}(\rho \varepsilon \boldsymbol{u})+\operatorname{div} \boldsymbol{q}_{T}=0, \quad \frac{\partial T}{\partial t}+\operatorname{div}(\boldsymbol{u} T)=a \nabla^{2} T, \quad a=\frac{\lambda}{\rho c_{p}} .
$$

The convection-diffusion (convection-heat conduction) equation is the basis of the mass transfer (heat transfer) theory. The basis of this equation is the intuition-judgment (axiom), that the velocity field and the concentration field (temperature) are the independent, i.e. the equation is linear.

There exist processes, where the mass transfer (heat transfer) induces secondary flows, i.e. the velocity field depends on the concentration (temperature) distributions and consequently the equation of convection-diffusion (convection-heat conduction) is a non-linear [3].

The non-linear mass transfer [3] is encountered in cases where secondary flows are induced by some physical effects, among them:

- large concentration gradient (non-linear mass transfer);

- surface tension gradient (Marangoni effect);

- vertical density gradient (natural convection);

- phase transition at the liquid-vapor phase boundary (Stephan flow).

In all these cases it is necessary to be used an additional law for the relationship between the secondary flow velocity and the concentration (temperature) distribution.

\section{Conclusion}

Some thoughts concerning the role of logic and the intuition in the chemical engineering allow formulating clearly their position in the modern science and practices. On this basis, to a greater extent, it is possible to elucidate some relationships between theories and experiments in the chemical engineering practice.

\section{References}

[1] Feinberg, E.L. (2004) Two Cultures. Intuition and Logic in the Art and Science. AGE 2, Fryazino, 286 p.

[2] Boyadjiev, C. (2010) Theoretical Chemical Engineering. Modeling and Simulation. Springer-Verlag, Berlin, Heidelberg, 594 p.

[3] Boyadjiev, C.B. and Babak, V.N. (2000) Non-Linear Mass Transfer and Hydrodynamic Stability. Elsevier, Amsterdam, New York, 500 p. 